\title{
STOCHASTIC RADIATIVE TRANSFER MODEL FOR MIXTURE OF DISCONTINUOUS VEGETATION CANOPIES
}

\author{
Nikolay V. Shabanov ${ }^{\mathrm{a}}$, D. Huang ${ }^{\mathrm{b}}$, Y. Knjazikhin ${ }^{\mathrm{a}}$, and Ranga B. Myneni ${ }^{\mathrm{a}}$ \\ åDepartment of Geography, Boston University, Boston, MA, 02215, USA - (shabanov, jknjazi, rmyneni)@bu.edu \\ ${ }^{\mathrm{b}}$ Brookhaven National Laboratory, Environmental Sciences Department, P.O. Box 5000, Upton, NY, 11973, USA - \\ dhuang@bnl.gov
}

KEY WORDS: Radiative Transfer, Theory, Three-domensional effects, vegetation structure, mixture modeling.

\begin{abstract}
:
Modeling of the radiation regime of a mixture of vegetation species is a fundamental problem of the Earth's land remote sensing and climate applications. The major existing approaches, including the linear mixture model and the turbid medium mixture Radiative Transfer model, provide only an approximate solution to this problem. In this study we developed the Stochastic Mixture Radiative Transfer (SMRT) model, a mathematically exact tool to evaluate radiation regime in a natural canopy with spatially varying optical properties (mixture of vegetation species and gaps). The model solves for the radiation quantities, direct input to the remote sensing/climate applications: mean radiation fluxes over whole mixture and over individual species. The canopy structure is parameterized in the SMRT model in terms of two stochastic moments: the probability of finding species and the conditional paircorrelation of species. We performed analytical and numerical analysis of the radiation effects, simulated with the SMRT model for the three cases of canopy structure: a) non-ordered mixture of species and gaps (turbid medium); b) ordered mixture of species without gaps; c) ordered mixture of species with gaps. The analysis indicates that the variation of radiation fluxes between different species is proportional to the variation of species optical properties. Gaps introduce significant disturbance to the radiation regime in the canopy as their optical properties constitute major contrast to those of any vegetation species. The SMRT model resolves deficiencies of the major existing mixture models: ignorance of species radiation coupling via multiple scattering of photons (the linear mixture model) or overestimation of this coupling due to neglecting spatial clumping of species (the turbid medium approach). Overall, this study establishes an advanced theoretical basis for future mixture applications.
\end{abstract}

\section{INTRODUCTION}

Natural vegetation exhibits significant degree of spatial heterogeneity, which complicates retrieval of the Earth's land biophysical parameters from remote sensing observations. Advances in remote sensing technology, including improved geolocation, sensor optics calibration, atmospheric correction, multi-resolution, multi-spectral and malti-angular measurements, etc., provide better means to capture land surface heterogeneity. In fact, current suite of NASA's MODerate resolution Imaging Spectroradiometer (MODIS) land products already includes a product, which explicitly characterizes mixture of land cover types- vegetation continuous fields (WWW1).

The problem of mixture of vegetation species is known in remote sensing as a scaling issue, that is, given biophysical parameters and radiation field over pure species at sub-pixel scale one needs to estimate those parameters at the scale of a mixed pixel. Multiple approaches were developed to address the scaling issue, which can be grouped into two basic categories: empirical/statistical and physically-based. The approaches from the first category are widely used for sub-pixel land cover characterization: linear mixture models (DeFries et al., 1999), neural networks (Carpenter et al., 1999), Gaussian mixture discriminant analysis, decision trees and others. The key idea of the above methods is to model satellite measured radiation over a mixed pixel as a weighted sum of the radiation fields over pure classes. Linear and non-linear models were implemented to retrieve the unknown weights, corresponding to the proportion of pure land cover classes in the mixed pixel. It was noted, however, that species in a mixture may exhibit significant degree of radiative interaction, which may bias retrievals especially in the case of linear models (Borel \& Gerstl, 1994).

In contrast to the empirical methods, physically-based approaches describe in details the physical processes of interaction of radiation with canopy at the level of elementary volume of vegetation. Optical properties of a mixture in such volume are represented as weighted average of optical properties of pure species. The Radiative Transfer Equation (RTE) is used to model the radiation field with effective optical properties of mixed canopy. The above modeling principles were implemented, for instance, in a scaling scheme of the radiation block of the Common Land Model (CLM) (WWW2, Tian et al., 2003) and the MODIS Leaf Area Index (LAI) algorithm (Tian et al., 2002). The major limitation of the above schemes is that they are based on the turbid medium mixture approximation, where canopy is represented as a mixed gas of vegetation species and gaps. With the turbid medium approach, one major feature of the natural vegetation is missing- spatial structure of a mixture, which may substantially affect radiation regime.

This study is aimed to advance theoretical description of the radiation regime in vegetation canopy under condition of spatial gradient of canopy optical properties. Applications in focus include the above mentioned radiation block of CLM and the MODIS LAI algorithm. The spatial heterogeneity of a medium can be incorporated in the standard RT equation using the stochastic approach, which was originally formulated for broken clouds by Vainikko (1973a,b) and further developed by Titov (1991) and others. Additional closely related theoretical 
study is the linear kinetic theory of stochastic mixture, developed by Pomraning (1991). In our former research (Shabanov et al., 2000, Huang et al, 2007) we adopted Vainikko-Titov approach for vegetation canopy and formulated the Stochastic RT (SRT) model for a single spatially discontinuous species. In the present work (Shabanov et al., 2007) we introduce a Stochastic Mixture RT (SMRT) model, an extension of the former model for the case of the structured composition of multiple vegetation species and gaps.

\section{THE APPROACH}

Consider 3D heterogeneous vegetation canopy, a mixture of $\mathrm{N}$ different vegetation species and gaps. The spatial structure of such canopy can be characterized by the indicator function of a canopy, $\chi(\vec{r})$, defined for each spatial location, $\vec{r}$, as follows:

$\chi(\overrightarrow{\mathrm{r}})=\sum_{\mathrm{j}} \chi^{(\mathrm{j})}(\overrightarrow{\mathrm{r}})$,

where $\chi^{(\mathrm{j})}(\overrightarrow{\mathrm{r}})$ is an indicator function of the individual species 'j':

$\chi^{(\mathrm{j})}(\overrightarrow{\mathrm{r}})= \begin{cases}1, & \text { if } \overrightarrow{\mathrm{r}} \in \text { species } \\ 0, & \text { otherwise. }\end{cases}$

The equations above specify overall architecture of vegetation canopy as cumulative contribution of individual species in a mixture. The indicator function is assumed to be a random variable. We further assume that a particular spatial location is occupied only by a single species, i.e.,

$\chi^{(\mathrm{i})}(\overrightarrow{\mathrm{r}}) \cdot \chi^{(\mathrm{j})}(\overrightarrow{\mathrm{r}})=0, \mathrm{i} \neq \mathrm{j}$.

Density of canopy is defined by the Leaf Area Index (LAI) one-sided green leaf area per unit ground area $\left[\mathrm{m}^{2} / \mathrm{m}^{2}\right]$. In the case of mixture of species,

$\mathrm{LAI}=\frac{1}{\mathrm{~S}} \int_{\mathrm{V}} \mathrm{d} \overrightarrow{\mathrm{r}} \mathrm{d}_{\mathrm{L}}(\overrightarrow{\mathrm{r}})=\sum_{\mathrm{j}} \frac{1}{\mathrm{~S}} \int_{\mathrm{V}} \mathrm{dr}_{\mathrm{r}} \mathrm{d}_{\mathrm{L}}^{(\mathrm{j})} \chi^{(\mathrm{j})}(\overrightarrow{\mathrm{r}})=\sum_{\mathrm{j}} \mathrm{LAI}^{(\mathrm{j})}$

where $\mathrm{d}_{\mathrm{L}}^{(\mathrm{j})}$ and $\mathrm{LAI}^{(\mathrm{j})}$ are one-sided foliage area volume density $\left[\mathrm{m}^{2} / \mathrm{m}^{3}\right]$ and LAI of species ' $\mathrm{j}$ ', respectively, and the integration is performed over a volume of canopy, $\mathrm{V}$, with a footprint, S. The interaction of radiation with species leaves is characterized by spatially varying extinction coefficient $\sigma(\vec{r}, \vec{\Omega})$ and differential scattering coefficient, $\sigma_{\mathrm{S}}\left(\overrightarrow{\mathrm{r}}, \vec{\Omega}^{\prime} \rightarrow \vec{\Omega}\right)$, (Ross, 1981),

$$
\begin{aligned}
& \sigma(\overrightarrow{\mathrm{r}}, \vec{\Omega})=\sum_{\mathrm{j}} \sigma^{(\mathrm{j})}(\vec{\Omega}) \chi^{(\mathrm{j})}(\overrightarrow{\mathrm{r}})=\sum_{\mathrm{j}} \mathrm{d}_{\mathrm{L}}^{(\mathrm{j})} \chi^{(\mathrm{j})}(\overrightarrow{\mathrm{r}}) \mathrm{G}^{(\mathrm{j})}(\vec{\Omega}), \\
& \begin{aligned}
\sigma_{\mathrm{S}}\left(\overrightarrow{\mathrm{r}}, \overrightarrow{\Omega^{\prime}} \rightarrow \vec{\Omega}\right) & =\sum_{\mathrm{j}} \sigma_{\mathrm{S}}^{(\mathrm{j})}\left(\vec{\Omega}^{\prime} \rightarrow \vec{\Omega}\right) \chi^{(\mathrm{j})}(\overrightarrow{\mathrm{r}}) \\
& =\sum_{\mathrm{j}} \frac{\mathrm{d}_{\mathrm{L}}^{(\mathrm{j})} \cdot \chi^{(\mathrm{j})}(\overrightarrow{\mathrm{r}})}{\pi} \cdot \Gamma^{(\mathrm{j})}\left(\vec{\Omega}^{\prime} \rightarrow \vec{\Omega}\right),
\end{aligned}
\end{aligned}
$$

where $G^{(j)}(\vec{\Omega})$ is the mean projection of leaf normals in the direction $\vec{\Omega}$ and $\Gamma^{(\mathrm{j})}\left(\vec{\Omega}^{\prime} \rightarrow \vec{\Omega}\right)$ is the area scattering phase function for species ' $\mathrm{j}$ ' (Ross, 1981). The above parameters depend on the probability density of leaf normal orientation, $\mathrm{g}_{\mathrm{L}}\left(\overrightarrow{\mathrm{r}}, \vec{\Omega}_{\mathrm{L}}\right),\left(\vec{\Omega}_{\mathrm{L}}\right.$ is a leaf normal direction) and the spectral leaf albedo, $\omega(\vec{r}, \lambda)$ ( $\lambda$ is a wavelength) (Ross, 1981). Given the set of structural and optical parameters, the radiation regime in a vegetation canopy is described by the following $3 \mathrm{D}$ transport equation for radiation intensity, $\mathrm{I}(\overrightarrow{\mathrm{r}}, \vec{\Omega})$ :

$$
\begin{aligned}
& \Omega \nabla \mathrm{I}(\overrightarrow{\mathrm{r}}, \vec{\Omega})+\sigma(\overrightarrow{\mathrm{r}}, \vec{\Omega}) \mathrm{I}(\overrightarrow{\mathrm{r}}, \vec{\Omega}) \\
& \quad=\int_{4 \pi} \mathrm{d} \vec{\Omega}^{\prime} \sigma_{\mathrm{S}}\left(\overrightarrow{\mathrm{r}}, \overrightarrow{\Omega^{\prime}} \rightarrow \vec{\Omega}\right) \mathrm{I}\left(\overrightarrow{\mathrm{r}}, \overrightarrow{\Omega^{\prime}}\right) .
\end{aligned}
$$

The unique solution of the Eq. (4) is specified by the boundary conditions.

The detailed evaluation of the 3D radiation field is computationally expensive and often unnecessary for multiple applications. Namely, in application to satellite remote sensing, radiation field, averaged over a pixel is required. The stochastic approach to the radiative transfer directly addresses the above problem, by averaging the 3D RT equation (Eq. (5)) over a horizontal plane. Thus, the average $1 \mathrm{D}$ equation for the average radiation intensities is obtained. The mathematical formulation of the stochastic RT equation requires two types of averages: (1) $\mathrm{U}^{(\mathrm{i})}(\mathrm{z}, \vec{\Omega})$, mean intensity over the portion of the horizontal plane at depth $z$, occupied by species 'i'; (2) $\bar{I}(z, \vec{\Omega})$, mean intensity over the total space of the horizontal plane at depth $\mathrm{z}$,

$$
\begin{aligned}
& \mathrm{U}^{(\mathrm{i})}(\mathrm{z}, \vec{\Omega})=\lim _{\mathrm{R} \rightarrow \infty} \frac{1}{\mathrm{~S}_{\mathrm{R}} \cap \mathrm{T}_{\mathrm{z}}^{(\mathrm{i})}} \iint_{\mathrm{S}_{\mathrm{R}}} \mathrm{dxdy} \chi^{(\mathrm{i})}(\mathrm{x}, \mathrm{y}, \mathrm{z}) \mathrm{I}(\mathrm{x}, \mathrm{y}, \mathrm{z}, \vec{\Omega}), \\
& \overline{\mathrm{I}}(\mathrm{z}, \vec{\Omega})=\lim _{\mathrm{R} \rightarrow \infty} \frac{1}{\mathrm{~S}_{\mathrm{R}}} \iint_{\mathrm{S}_{\mathrm{R}}} \mathrm{dx} \mathrm{dy} \mathrm{I}(\mathrm{x}, \mathrm{y}, \mathrm{z}, \vec{\Omega}) .
\end{aligned}
$$

In the above, $\mathrm{S}_{\mathrm{R}}$ denotes the area of a circle of radius $\mathrm{R}$; $\mathrm{T}_{\mathrm{z}}^{(\mathrm{i})}$ denotes the area of the horizontal plane at depth $\mathrm{z}$, occupied by species ' $i$ '. In the framework of stochastic mixture, gaps can be treated as special type of vegetation species, optical properties of which are set to zero. Thus, in the case of $\mathrm{N}$ vegetation species we have $\mathrm{U}^{(\mathrm{i})}(\mathrm{z}, \vec{\Omega}), 1=1, \mathrm{~N}$ and $\mathrm{U}^{(\mathrm{gap})}(\mathrm{z}, \vec{\Omega})$.

The averaging procedure results in the parameterization of the resulting transfer equation in terms of two stochastic moments of a vegetation structure. The first stochastic moment is the probability, p, of finding species “i” at canopy depth z,

$\mathrm{p}^{(\mathrm{i})}(\mathrm{z})=\lim _{\mathrm{R} \rightarrow \infty} \frac{1}{\mathrm{~S}_{\mathrm{R}}} \underset{\mathrm{S}_{\mathrm{R}} \cap \mathrm{T}_{\mathrm{z}}^{(\mathrm{i})}}{\mathrm{i})}(\mathrm{z}, \mathrm{x}, \mathrm{y}) \mathrm{dxdy} \equiv \lim _{\mathrm{R} \rightarrow \infty} \frac{\mathrm{S}_{\mathrm{R}} \cap \mathrm{T}_{\mathrm{z}}^{(\mathrm{i})}}{\mathrm{S}_{\mathrm{R}}}$.

The second moment is the pair-correlation function, q, between species 'i' at canopy depth $\mathrm{z}$ and species 'j' at depth $\xi$ along the direction $\vec{\Omega}$,

$\mathrm{q}^{(\mathrm{i}, \mathrm{j})}(\mathrm{z}, \xi, \vec{\Omega})=\lim _{\mathrm{R} \rightarrow \infty} \frac{\mathrm{S}_{\mathrm{R}} \cap \mathrm{T}_{\mathrm{z}}^{(\mathrm{i})} \cap \mathrm{T}_{\xi}^{(\mathrm{j})}[\Delta \overrightarrow{\mathrm{r}}]}{\mathrm{S}_{\mathrm{R}}}$,

where

$\Delta \overrightarrow{\mathrm{r}}=\frac{\Omega_{\mathrm{x}}}{\Omega_{\mathrm{z}}}(\mathrm{z}-\xi), \frac{\Omega_{\mathrm{y}}}{\Omega_{\mathrm{z}}}(\mathrm{z}-\xi)$

and $\Omega_{\mathrm{x}}, \Omega_{\mathrm{y}}$, and $\Omega_{\mathrm{z}}$ are projections of a unit direction 
vector, $\vec{\Omega}$, on the $\mathrm{x}, \mathrm{y}$, and $\mathrm{z}$ axes, respectively. Argument for $\mathrm{T}_{\xi}^{(\mathrm{j})}$ denotes a shift of the origin of plane $\xi$ relative to plane $\mathrm{z}$ along $\mathrm{x}$ and $\mathrm{y}$ directions, required to evaluate correlation between the planes in direction $\vec{\Omega}$. Using the first and second moments of a vegetation structure, the conditional paircorrelation of species, $\mathrm{K}^{(\mathrm{i}, \mathrm{j})}$, can be evaluated as

$\mathrm{K}^{(\mathrm{i}, \mathrm{j})}(\mathrm{z}, \xi, \vec{\Omega})=\frac{\mathrm{q}^{(\mathrm{i}, \mathrm{j})}(\mathrm{z}, \xi, \vec{\Omega})}{\mathrm{p}^{(\mathrm{i})}(\mathrm{z})}$.

The two stochastic moments are key parameters of the stochastic approach, responsible for 3D radiation effects arising from non-homogeniety (3D structure) of vegetation canopy. The amount of gaps is controlled by the probability of finding species $\mathrm{p}^{(\mathrm{i})}$ (Eq. 3), while canopy structure (order/chaoticity) is controlled by the conditional pair-correlation of species, $\mathrm{K}^{(\mathrm{i}, \mathrm{j})}$, (Eq. 5).
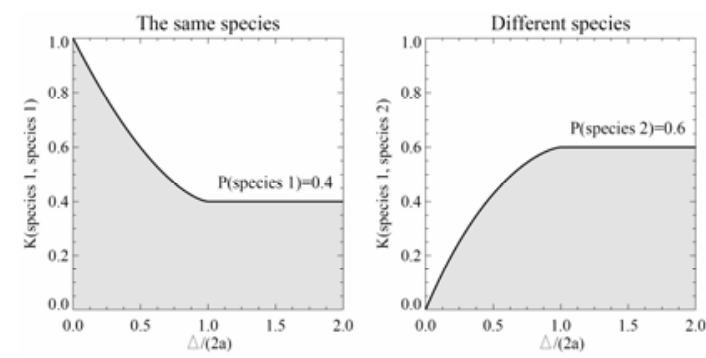

Figure 1: The conditional pair-correlation function, $\mathrm{K}^{(\mathrm{i}, \mathrm{j})}$, of the SMRT model as function of horizontal distance of correlation, $\Delta$, normalized by three diameter, 2a. The parameters are as follows: two species with probabilities $\mathrm{p}^{(1)}=0.4$ and $\mathrm{p}^{(2)}=0.6$; tree radius $\mathrm{a}=0.15$; canopy height $\mathrm{H}=1$.

The conditional pair-correlation function can be derived according to the theory of stochastic geometry (Stoyan, Kendall, Mecke, 1995). This approach was utilized recently to derive the conditional pair-correlation function for single species (Huang et al., 2007). In this research we extended derivations to a mixture of multiple species (Shabanov et al., 2007). The derivations are based on the following assumptions about 3D stochastic canopy structure: a) tree species are modeled as identical cylinders; b) distribution of the tree centers follows stationary Poisson point process (Stoyan, Kendall, Mecke, 1995). The conditional pair-correlation function for two species is shown in Fig. 1. In the case of the same species, correlation decreases as distance increases. This corresponds to increasing probability of one of the points being out of the same crown as distance increases. In the case of different species, correlation increases as distance increases. This corresponds to increasing probability of two points to be located in different crowns of different species with increasing distance. In the case of short distances, within-species correlation is 1 , while between-species correlation is 0 . In the case of large distances, correlation between any species is vanishing and $\mathrm{K}^{(\mathrm{i}, \mathrm{j})}$ converges to $\mathrm{p}^{(\mathrm{i})}$. Both limiting cases are intuitively expected and captured by the proposed model.

\section{ANALYSIS}

The SMRT equations were derived and implemented numerically using Successive Orders of Scattering
Approximations (SOSA) (Shabanov et al., 2007). The analytical analysis of the SMRT model is beyond the scope of this paper and details can be found in Shabanov et al., (2007). Here we review some results of numerical analysis of 3D radiation effects, resulting from 3D canopy structure. We simulated three key classes of canopy structure as captured by stochastic moments of structure: a) non-ordered/chaotic mixture of species and gaps or turbid medium $\left(\mathrm{K}^{(\mathrm{i}, \mathrm{j})}=\mathrm{p}^{(\mathrm{j})}\right)$; b) ordered mixture of species without gaps $\left(\sum_{j} p^{(j)}=1\right)$; c) ordered mixture of species with gaps $\left(\mathrm{K}^{(\mathrm{i}, \mathrm{j})} \neq \mathrm{p}^{(\mathrm{j})}, \sum_{\mathrm{j}} \mathrm{p}^{(\mathrm{j})} \neq 1\right)$. In the analysis below, we contrast the SMRT simulations under condition of canopy structure ("SMRT", cases b and c) with turbid medium model simulations ("TM", case a). Both cases were implemented with the same set of stochastic equations and input parameters, except the conditional pair-correlation function: the analytical expression for cylindrical trees (Shabanov et al., 2007) was used to implement the SMRT model, while $\mathrm{K}^{(\mathrm{i}, \mathrm{j})}=\mathrm{p}^{(\mathrm{j})}$ was used for the TM model.
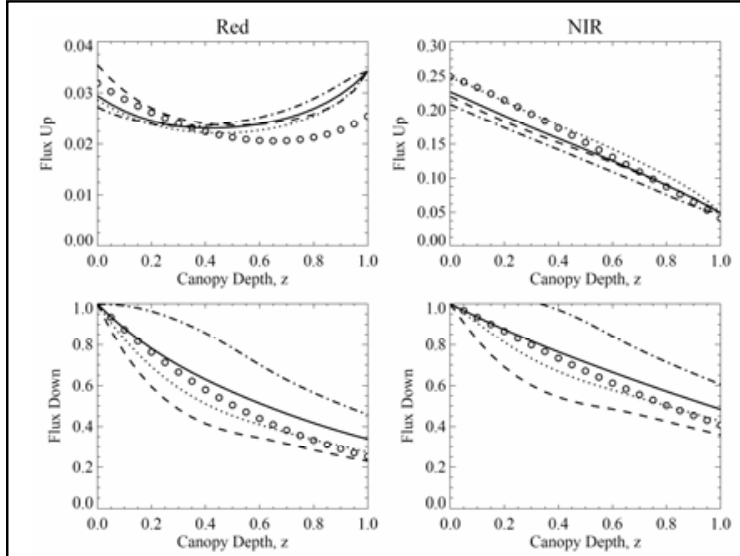

Figure 2: The Comparison of vertical profiles of up and down radiation fluxes as simulated by the TM and SMRT models for mixture of two species and gaps. The SMRT model captures spatial variation of fluxes between species 1 (dashed line), species 2 (dotted line), and gaps (dash-dot line) and also evaluates average flux over whole mixture (solid line). The TM model estimates only average flux over whole mixture (hollow dots). The models parameters are as follows: $\mathrm{p}^{(1)}=0.40, \mathrm{p}^{(2)}=0.20 ; \mathrm{d}_{\mathrm{L}}^{(1)}=4, \mathrm{~d}_{\mathrm{L}}^{(2)}=6$; $\omega^{(1)}($ Red $)=0.12, \omega^{(2)}($ Red $)=0.20, \omega^{(1)}(\mathrm{NIR})=0.90$, $\omega^{(2)}(\mathrm{NIR})=0.60 ; \rho_{\text {soil }}(\mathrm{Red})=\rho_{\text {soil }}(\mathrm{NIR})=0.10$; direct incoming flux, $\mathrm{SZA}=15^{0}$.

To understand the overall merits of the new model consider radiation fluxes as function of canopy depth, as shown in Fig. 2. The SMRT model differentiates between radiation fluxes over individual species, gaps, and whole mixture (corresponding to mean intensities $\mathrm{U}^{(\mathrm{i})}, \mathrm{U}^{(\mathrm{gap})}, \overline{\mathrm{I}}$ ). The TM model provides no distinction between the above fluxes. According to the SMRT model, variation of the fluxes between individual vegetation species is relatively smaller compared to difference in fluxes between vegetation and gaps. Also note, that limitations of the TM model result in a bias (both overestimation and underestimation) in estimation of mean fluxes over whole mixture compared to the SMRT simulations (compare fluxes over whole mixture). Detailed analysis of the impact of various parameters on the SMRT and TM models simulations is presented with four case studies below (Figs. 3-6). 


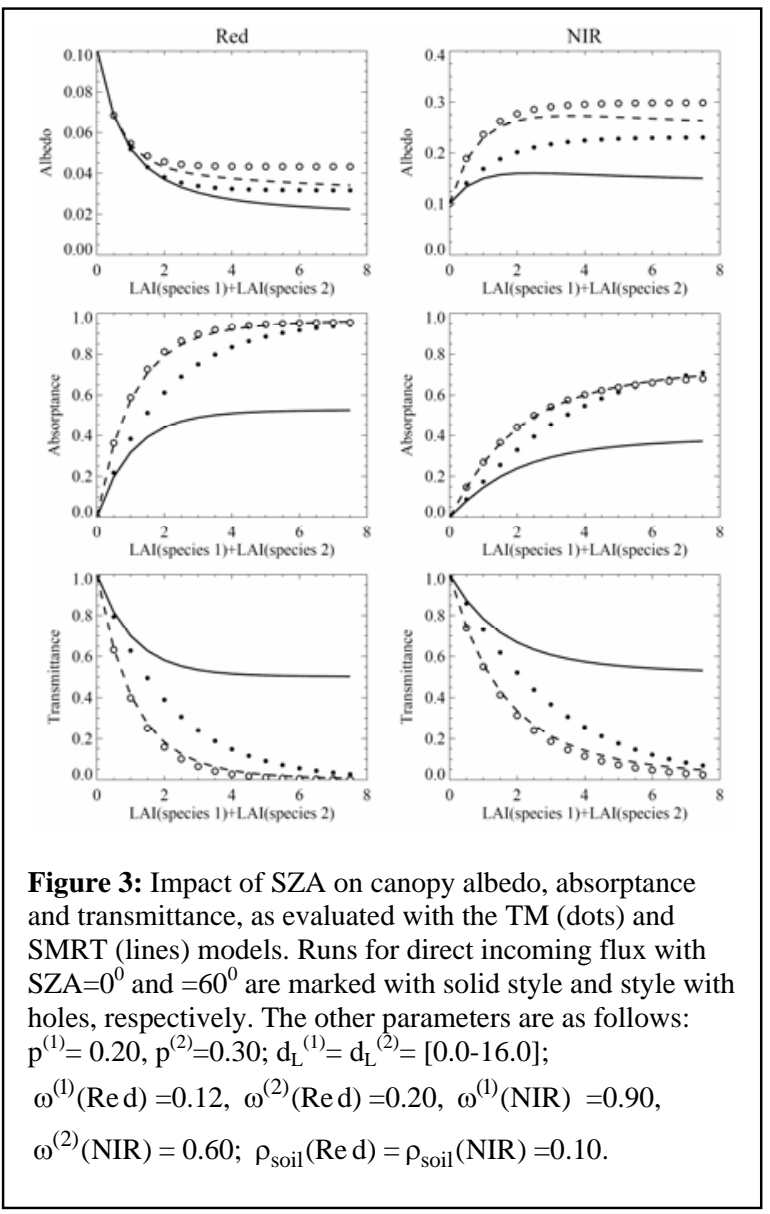

First, consider impact of Solar Zenith Angle (SZA) on canopy albedo, absorptance and transmittance as function of LAI, as simulated with the SMRT and TM models at Red and NIR wavelengths (Fig. 3). The simulations were performed with SZA of $0^{\circ}$ and $60^{\circ}$. Two vegetation species with gaps were used: $\mathrm{p}^{(1)}=0.2, \quad \mathrm{p}^{(2)}=0.3, \quad \mathrm{p}^{\text {(gap) }}=0.5$. The complete set of parameters is presented in the figure caption. In the case of simulations for $\mathrm{SZA}=0^{\circ}$, the SMRT model predicts lower albedo, substantially lower absorptance and substantially higher transmittance compared to the TM model. However, at $\mathrm{SZA}=60^{\circ}$ both models demonstrate quite similar results. The key physical explanation for the difference between the SMRT and TM simulations at $\mathrm{SZA}=0^{0}$ is that the SMRT model accounts for radiation streaming through gaps without interaction with vegetation. This explains results for absorptance and transmittance. Albedo is lower in the case of the SMRT compared to the TM model, because in the former case a dark soil is better exposed through gaps in a relatively bright vegetation (compare $\rho_{\text {soil }}$ and $\omega$ ). Note the difference in models prediction for a limiting case of high LAI (cf. case for Red wavelength, SZA $=0^{\circ}$ ). In the case of the SMRT model, canopy transmittance for high LAI approaches to 0.5, which corresponds to radiation streaming through gaps in the amount of $1-\left(\mathrm{p}^{(1)}+\mathrm{p}^{(2)}\right)$. In contrast, in the case of the TM model, transmittance converges to 0 , which is a consequence of lack of gaps in the TM model formulation. Next, we explain results for $\mathrm{SZA}=60^{\circ}$. In this case the effect of radiation streaming is negligible: even if photon enters canopy through a gap, it will be intercepted by a lateral surface of tree foliage. Mathematically, the reasoning is as follows: as angle is increasing, effective distance between vegetation elements, $\Delta$, is increasing, which results in convergence of the conditional pair-correlation function for ordered species to one for nonordered species (cf. Fig 1).

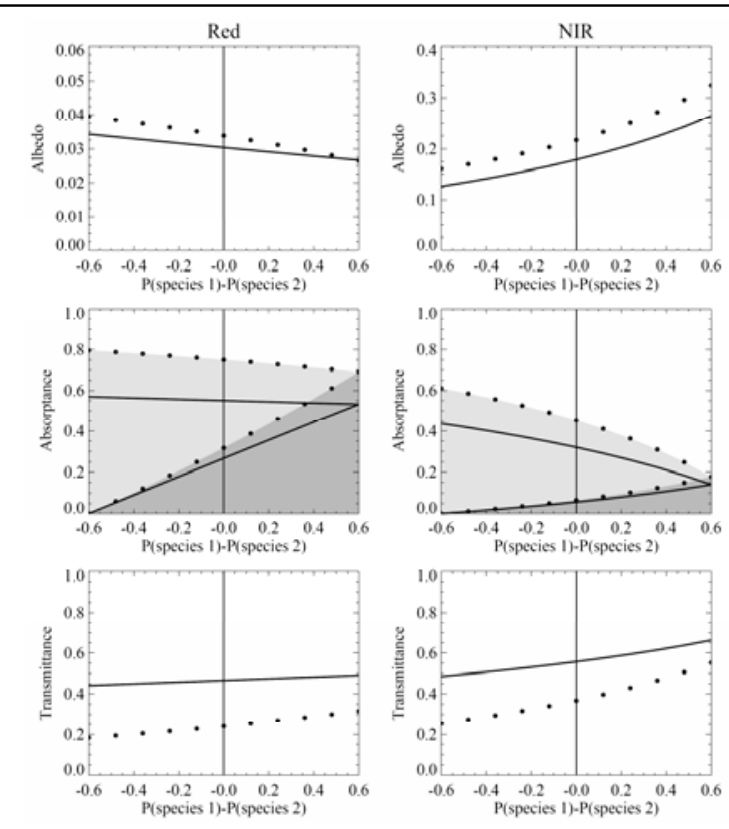

Figure 4: Impact of species composition on canopy albedo, absorptance and transmittance, as evaluated with the TM (dots) and SMRT (lines) models for the case of mixture of two species. The models parameters are as follows:

$\mathrm{p}^{(1)}+\mathrm{p}^{(2)}=0.60 ; \mathrm{d}_{\mathrm{L}}^{(1)}=4.0, \mathrm{~d}_{\mathrm{L}}^{(2)}=6.0 ; \omega^{(1)}(\operatorname{Red})=0.12$,

$\omega^{(2)}(\mathrm{Red})=0.20, \omega^{(1)}(\mathrm{NIR})=0.90, \omega^{(2)}(\mathrm{NIR})=0.60$;

$\rho_{\text {soil }}($ Red $)=\rho_{\text {soil }}($ NIR $)=0.10$; direct incoming flux,

$\mathrm{SZA}=0^{0}$. Shaded areas highlight absorptance of individual species in the case of the TM model simulations.

Second, consider the impact of species composition on a radiation regime (Fig. 4). Here we used two species with optical properties roughly corresponding to broadleaf (species 1) and needle leaf (species 2) forests, which have substantial contrast both at Red and NIR wavelengths. The probability of each species $\left(\mathrm{p}^{(1)}\right.$ and $\left.\mathrm{p}^{(2)}\right)$ was varying from 0 up to 0.6 , under restriction, that total probability of all species is constant through the simulations, i.e., $\mathrm{p}^{(1)}+\mathrm{p}^{(2)}=0.6=$ fixed. Thus, radiation regime was evaluated for all possible combinations of two species under significant amount of gaps, $\mathrm{p}^{\text {(gap) }}=0.4=$ fixed. Our results indicate that both the TM and SMRT models simulate continuous, fairly large variation of canopy albedo, absorptance and transmittance with respect to species composition. However, the TM model introduces a significant bias in the estimation of the above parameters due to ignoring canopy structure. Finally, note that variations of canopy albedo, absorptance and transmittance with respect to species compositions are quite close to linear at Red wavelength, but demonstrate fairly large deviation from linearity at NIR wavelength.

In the third case study we probed deeper special case of mixture of species without gaps (cf. Fig. 5). Intuitively, in the case of no gaps, ordered mixture of species is quite close to non-ordered mixture (turbid medium). In this simulation we addressed two 
questions: a) When ordered mixture of species is important? b) Why gaps significantly perturb radiation field of ordered mixture of species? As mentioned earlier, key feature of vegetation structure, which differentiates between ordered and non-ordered cases is a presence of spatial gradient of optical properties of a medium (such as leaf albedo, density of LAI, etc). If the medium is ordered, the spatial gradient of the optical properties should be significant enough to modify the radiation regime of the SMRT simulations with respect to the TM simulations. Optical properties of gaps constitute especially large contrast to ones of any vegetation species. This explains special role of gaps in the SMRT simulations. The variation of the optical properties of vegetation species is as follows: leaf albedo may vary by factor of 2 in majority of cases, while variation in the foliage area volume density may be arbitrary large. Our test runs (not presented here) indicate no significant difference between the SMRT and TM simulations as function of variations of leaf albedo for typical vegetation canopies. Simulations for two species with varying foliage area volume density $\left(\mathrm{d}_{\mathrm{L}}{ }^{(1)}\right.$ and $\left.\mathrm{d}_{\mathrm{L}}{ }^{(2)}\right)$ indicate significant bias in the estimation of absorptance by the TM model with respect to the SMRT model when $\mathrm{d}_{\mathrm{L}}^{(2)} / \mathrm{d}_{\mathrm{L}}^{(1)}=0$, no difference when $\mathrm{d}_{\mathrm{L}}^{(2)} / \mathrm{d}_{\mathrm{L}}{ }^{(1)}=1$, and increasing bias with respect to increasing contrast in the foliage area volume density of species (Fig. 5). Note that the TM model introduces bias not only to total absorptance but also to the partitioning of total canopy absorptance between species.
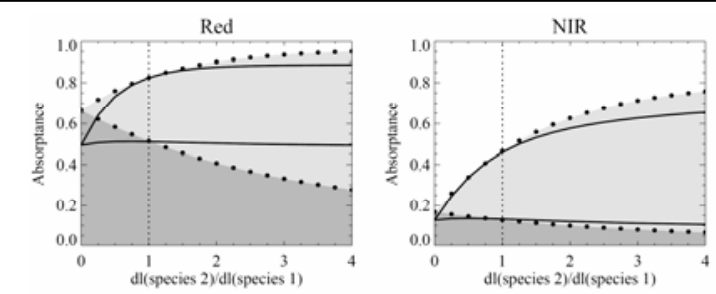

Figure 5: Impact of species LAI density on partitioning of total absorptance between individual species, as evaluated with the TM (dots) and SMRT (lines) models for the case of mixture of two species. The models parameters are as follows: $\mathrm{p}^{(1)}=0.4, \mathrm{p}^{(2)}=0.6 ; \mathrm{d}_{\mathrm{L}}^{(1)}=4.0, \mathrm{~d}_{\mathrm{L}}^{(2)}=[0.0-16.0]$;

$\omega^{(1)}(\operatorname{Red})=0.12, \omega^{(2)}(\operatorname{Red})=0.20, \omega^{(1)}(\mathrm{NIR})=0.90$,

$\omega^{(2)}(\mathrm{NIR})=0.60 ; \rho_{\text {soil }}(\operatorname{Red})=\rho_{\text {soil }}(\mathrm{NIR})=0.10 ;$ direct

incoming flux, $\mathrm{SZA}=0^{0}$. Shaded areas highlight

absorptance of individual species in the case of the TM

model simulations.

Next, consider the fourth case study, demonstrating the effect of species interaction (Fig. 6). Here we evaluated the impact of LAI changes of species 2 on the absorptance of species 1 . The LAI of species 2 was constructed according to two scenarios: a) keep $\mathrm{p}^{(2)}$ constant and vary $\mathrm{d}_{\mathrm{L}}{ }^{(2)}$ (top two panels for Red and NIR wavelengths); b) keep $d_{L}^{(2)}$ constant and vary $\mathrm{p}^{(2)}$ (lower panels). We also used two illumination conditions: purely direct incoming flux, $\mathrm{SZA}=0^{0}$; and purely diffuse incoming flux. The results indicate, that absorptance of species 1 decreases by factor of two both at Red and NIR wavelengths as LAI of species 2 is changing from 0 to 4 in the case of the TM model under direct illumination. In contrast, the SMRT model predicts no significant variations in the absorptance of species 1 at Red wavelength, and increase by about $15 \%$ in the case of NIR wavelength under direct illumination. In the case of diffuse illumination the SMRT and TM models predict similar interaction of species- decrease of the absorptance of the first species as LAI of the second is increasing. This last result of this case study match the results of the first case study: under diffuse illumination or low SZA, the simulations by both models converge. Overall, this last case study demonstrates that natural mixture of discontinuous species exhibit less radiative coupling compared to the turbid medium approximation.
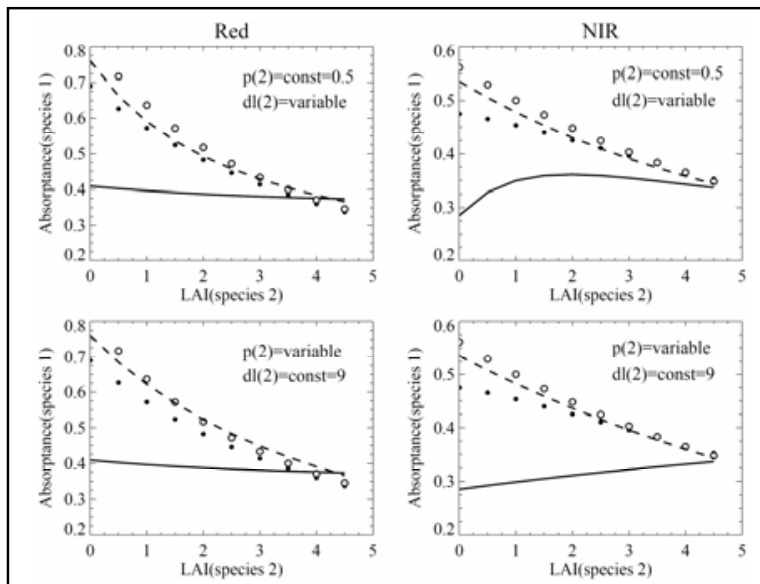

Figure 6: Impact of species interaction on canopy absorptance, as evaluated with the TM (dots) and SMRT (lines) models. Runs for direct $\left(\mathrm{SZA}=0^{0}\right)$, and diffuse incoming fluxes are marked with solid style and style with holes, respectively. The other parameters are as follows: $\mathrm{p}^{(1)}=0.4, \mathrm{p}^{(2)}=0.5$ or variable; $\mathrm{d}_{\mathrm{L}}^{(1)}=6.0, \mathrm{~d}_{\mathrm{L}}{ }^{(2)}=9.0$ or variable; $\omega^{(1)}(\operatorname{Red})=0.12, \omega^{(2)}(\operatorname{Red})=0.16, \omega^{(1)}(\mathrm{NIR})$ $=0.60, \omega^{(2)}(\mathrm{NIR})=0.80 ; \rho_{\text {soil }}(\operatorname{Red})=\rho_{\text {soil }}(\mathrm{NIR})=0.10$.

The above presented analysis was focused on comparison of the SMRT and turbid medium models. Here we briefly comment on the performance of the linear mixture model. Multiple land algorithms utilized for estimation of land cover mixture from coarse resolution satellite data rely on the empirical model of linear mixture of species (cf. Section 1). Under this assumption canopy spectral reflectance of a mixed pixel is expressed as a linear combination of canopy spectral reflectances of pure species (DeFries et al., 1999). The linear mixture model ignores species radiative coupling. This coupling in a natural canopy is caused by multiple scattering, that is, after interaction with phytoelements of the first species, photon is scattered into another species. The SMRT model can be reduced to the linear mixture model, and allows analysis of empirical assumptions of the latter. According to stochastic equations (Shabanov et al., 2007), mean radiation over mixed pixel, $\overline{\mathrm{I}}(\mathrm{z}, \vec{\Omega})$, is equal to a weighted average of the radiation fields over pure species, $\mathrm{U}^{(\mathrm{i})}(\mathrm{z}, \vec{\Omega})$, and gaps, $\mathrm{U}^{(\mathrm{gap})}(\mathrm{z}, \vec{\Omega})$. However, $\mathrm{U}^{(\mathrm{i})}(\mathrm{z}, \vec{\Omega})$ are coupled though system of stochastic equations. In order to derive the linear mixture model from SMRT model one needs to break the coupling, that is, set to 0 the conditional paircorrelation function for different species, $\mathrm{K}^{(\mathrm{i}, \mathrm{j})}=0$, when $\mathrm{i} \neq \mathrm{j}$. This assumption apparently violates the basic geometry constraints on $\mathrm{K}^{(\mathrm{i}, \mathrm{j})}$, however the corresponding stochastic equations are mathematically correct and energy conservation law is preserved in this case as shown by Shabanov et al. (2007). Therefore, while radiative decoupling of vegetation species is physically meaningless, it is still a mathematically valid exercise and may describe RT processes in some other 
medium. Overall, our results indicate that the linear mixture model ignores, while turbid medium overestimates species radiative coupling compared to a realistic description of the SMRT model.

\section{CONCLUSIONS}

In this research we developed the Stochastic Mixture RT (SMRT) model for simulation of radiation regime in a natural vegetation canopy with spatially varying optical properties. The new approach provides a general solution of the problem, which includes, as special cases, the major approximate solutions, including the linear mixture and turbid medium mixture RT models. The SMRT model solves for the radiation quantities, direct input to remote sensing/climate applications: mean fluxes over mixture and over individual species. The canopy structure is parameterized in the SMRT model in terms of two stochastic moments: the probability of finding species and the conditional pair-correlation of species. The second moment is responsible for the $3 \mathrm{D}$ radiation effects, namely, radiation streaming through gaps without interaction with vegetation and variation of the radiation fluxes between different species. If the within- and between- species correlation is vanishing, the SMRT model reduces to the turbid medium RT model. Namely, this situation is realized in the SMRT simulations under direct illumination with low SZA or diffuse illumination. If the between- (but not within-) species correlation is set to zero, the SMRT model reduces to the linear mixture model. The analysis of the SMRT simulations indicates that the variation of radiation fluxes between different species is proportional to the variation of optical properties of species (leaf albedo, density of foliage, etc.) Gaps introduce significant disturbance to the radiation regime in the mixed canopy as their optical properties constitute a major contrast to those of any vegetation species. Set of accurate field measurements on canopy structure and radiation is required to further assess performance of the SMRT model and to improve modeling of the pair-correlation function.

\section{ACKNOWLEDGMENTS}

This research was supported by NASA MODIS contract NNG04HZ09C and Interdisciplinary Science (IDS) in the NASA Earth Science Enterprise grant G35C14G2.

\section{REFERENCES}

WWW1: Vegetation continuous fields product from MODIS, http://glcf.umiacs.umd.edu/data/modis/vcf/

WWW2: Common Land Model (CLM), http://www.cgd.ucar.edu/tss/clm/distribution/clm3.0/index.html

Borel C., Gerstl S., 1994. Nonlinear spectral mixing models for vegetative and soil surfaces. Remote Sensing of Environment, 47, pp. 403-416.

Carpenter G.A., Gopal S., Macomber S., Martens S., Woodcock C.E., 1999. A neural network method for mixture estimation for vegetation mapping. Remote Sensing of Environment, 70, pp. 135-152.

DeFries R.S., Townshend J.R.G., Hansen M.C., 1999. Continuous fields of vegetation characteristics at the global scale 1-km resolution. Journal of Geophysical ResearchAtmosphere, 104(D14), pp. 16911-16923.

Huang D., Knyazikhin Y., Yang W., Wang W., Privette J., Deering D., Shabanov N., Myneni R.B., 2007. Stochastic transport theory for investigating the three-dimensional canopy structure from space measurements. Remote Sensing of Environment, accepted, June 2006.

Pomraning G.C., 1991. Linear kinetic theory and particle transport in stochastic mixtures. Series on advances in mathematics for applied sciences.-Vol. 7. Singapore: World Scientific.

Ross J., 1981. The radiation regime and architecture of plant stands. Hague: Dr. W. Junk Publishers.

Shabanov N.V., Knyazikhin Y, Baret F, Myneni RB., 2000. Stochastic modeling of radiation regime in discontinuous vegetation canopies. Remote Sensing of Environment, 74(1), pp. 125-144.

Shabanov N.V., Huang, D., Knyazikhin Y, Dickinson, R.E., Myneni RB., 2007. Stochastic Radiative Transfer Model for Mixture of Discontinous Vegetation Canopies. Journal of Quantitative Spectroscopy and Radiative Transfer. In press

Stoyan D, Kendall W.S., Mecke J., 1995. Stochastic geometry and its applications. Wiley Series in Probability and Statistics, Second Ed., John Wiley \& Sons, pp.67-69.

Tian Y., Wang Y., Zhang Y., Knyazikhin Y., Bogaert J., Myneni, R.B., 2002. Radiative transfer based scaling of LAI/FPAR retrievals from reflectance data of different resolutions. Remote Sensing of Environment, 84, pp. 143-159.

Tian Y., Dickinson R.E., Zhou L., Zeng X., Dai Y., Myneni R.B., Knyazikhin Y., Zhang X., Friedl M., Yu H., Wu W., Shaikh M., 2004. Comparison of seasonal and spatial variations of LAI/FPAR from MODIS and Common Land Model. Journal of Geophysical Research-Atmosphere, 109(D1), D01103, 10.1029 /2003JD003777.

Titov, G.A., 1990. Statistical description of radiation transfer in clouds. Journal of the Atmospheric Sciences, 47, pp. 24-38.

Vainikko, G.M., 1973a. The equation of mean radiance in broken cloudiness. Trudy MGK SSSR, Meteorological investigations, 21, pp. 28-37, (in Russian).

Vainikko, G.M., 1973b. Transfer approach to the mean intensity of radiation in non-continuous clouds. Trudy MGK SSSR, Meteorological investigations, 21, pp. 38-57, (in Russian). 